Cipango Cahiers d'études japonaises

17 | 2010

La péninsule retrouvée

Jacqueline PIGEOT, Michiyuki-bun, poétique de l'itinéraire dans la littérature du Japon ancien

Sylvie Brosseau

(2) OpenEdition

Journals

Édition électronique

URL : https://journals.openedition.org/cipango/1134

DOI : 10.4000/cipango. 1134

ISSN : 2260-7706

Éditeur

INALCO

Édition imprimée

Date de publication : 30 juin 2010

Pagination : 247-261

ISBN : 978-2-85831-195-8

ISSN : 1164-5857

Référence électronique

Sylvie Brosseau, « Jacqueline pıgeot, Michiyuki-bun, poétique de l'itinéraire dans la littérature du Japon ancien », Cipango [En ligne], 17 | 2010, mis en ligne le 16 novembre 2012, consulté le 30 juin 2021. URL : http://journals.openedition.org/cipango/1134; DOI : https://doi.org/10.4000/cipango.1134

Ce document a été généré automatiquement le 30 juin 2021.

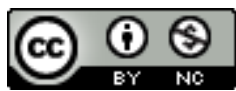

Cipango est mis à disposition selon les termes de la Licence Creative Commons Attribution - Pas d'Utilisation Commerciale 4.0 International. 


\title{
Jacqueline PIGEOT, Michiyuki-bun, poétique de l'itinéraire dans la littérature du Japon ancien
}

\author{
Sylvie Brosseau
}

\section{RÉFÉRENCE}

Jacqueline PIGEOT, Michiyuki-bun, poétique de l'itinéraire dans la littérature du Japon ancien, Ed. revue et corrigée, Bibliothèque de l'Institut des Hautes Études Japonaises, Collège de France, 2009, 410 p. ISBN : 978-2-913217-21-8

C'est avec un intérêt renouvelé que nous suivons les parcours ouverts par Jacqueline Pigeot grâce à une deuxième édition bienvenue de son ouvrage Michiyuki-bun, doté d'un sous-titre explicite Poétique de l'itinéraire dans la littérature du Japon ancien. Cette stimulante étude littéraire part en quête de la genèse d'un genre, le michiyuki-bun 道行 文, forme de relation d'itinéraire, et analyse l'élaboration d'un langage poétique. Sa lecture enrichit amplement les connaissances dans les champs de la perception et de la conception du territoire, des sites, des paysages. Ces approches utiles à l'étude de la spatialité au Japon confirment l'importance des recherches sur la littérature classique pour une meilleure et plus juste compréhension de la culture japonaise.

La présente réédition est modestement introduite par l'auteure comme une révision. Des annexes abordant des thèmes traités dans ses publications postérieures ont été supprimées, des corrections, apportées et des notes, allégées. Outre cette relecture, ont été intégrées environ vingt nouvelles références d'ouvrages (français, anglais, japonais) publiés depuis la première édition en 1982. Plusieurs débuts ou fins de chapitres, donc des articulations de l'ensemble, ont été réécrits, particulièrement la quatrième et dernière partie du livre, analyse thématique inspirée et suggestive. Beaucoup de poèmes sont retraduits, d'autres ajoutés. De ce minutieux travail, ressort un recentrage sur la thématique de l'itinéraire et du voyage traités par la généalogie de leurs 
expressions littéraires, l'évolution des poétiques spécifiques à divers genres dont le michiyuki-bun est considéré comme une forme d'aboutissement.

Une nouvelle annexe sur «La vie quotidienne des voyageurs à l'époque médiévale » met en relief une constante fondamentale. Le territoire du Japon est, depuis fort longtemps, parcouru par de nombreux voyageurs qui vont et viennent entre les différentes capitales et les provinces pour de multiples raisons. S'ajoutent aux voyages officiels ou d'agrément de l'aristocratie, déplacements des fonctionnaires entre la capitale et leur poste, mouvements des escortes militaires, approvisionnements depuis la campagne, déplacements de main-d'œuvre, pèlerinages de toutes sortes, pérégrinations de religieux, colporteurs ou divers artistes itinérants, errances des plus pauvres. Les axes principaux centrés sur la capitale croisent les routes de pèlerinage et les voies d'eau, fluviales ou maritimes. Pour organiser et contrôler ces déplacements incessants - les "gens du voyage " représentent toujours et partout une menace - les premières règlementations, sans doute peu suivies, étonnent par leur ancienneté puisqu'un système de relais (eki 駅) est répertorié en 646. Également fort ancien, un édit de 759 (rédigé par un moine de Nara sur le modèle chinois) préconise de planter le long des routes des arbres fruitiers (pêchers et poiriers nous semble-t-il) afin d'améliorer les conditions de voyage, surtout des plus pauvres. Jacqueline Pigeot note que ce règlement ne sera sans doute pas suivi d'application (du moins à grande échelle) mais des édits de ce type seront sans cesse réitérés, jusqu'à ce que, plus tard, une administration soit chargée de l'entretien des routes et des plantations d'alignement très diversifiées. À partir du XII siècle, l'hébergement des voyageurs commence à s'organiser dans les bourgs étapes, le long des routes comme dans les sites de pèlerinage. Malgré tout, voyager demeure une expérience dangereuse qui requiert de s'assurer la protection des divinités, tels les Dosō-jin 道祖神, par des offrandes et des cultes, d'où les stèles jalonnant les chemins, les carrefours ou les passages difficultueux. Bien plus tard, l'unification du pays favorisera parmi toutes les classes de la population un essor des voyages qui tendront vers des formes de tourisme. Cependant, au préalable, nombre de sites et hauts lieux sont réellement parcourus, connus et reconnus par «d'infatigables poètes» comme par d'innombrables voyageurs anonymes.

4 Le michiyuki-bun, appellation moderne d'une forme littéraire créée à l'époque de Kamakura, est dans un récit épique, un passage où une suite de toponymes forme un itinéraire parcouru. Les sentiments du voyageur sont suggérés et confèrent une tonalité affective ; les paysages des sites traversés sont brièvement évoqués, suivant des conventions dont l'élaboration stylistique fait l'objet de la recherche. Si ce voyage est fictif, les étapes se situent sur des routes bien réelles, parmi les plus fréquentées. Cette relation rhétorique d'itinéraires exprime à la fois progression temporelle et continuité spatiale, créant une impression de glissement par l'enchaînement des noms de lieux. L'étude de Jacqueline Pigeot croise deux types d'approches, d'une part l'histoire d'une forme littéraire, à travers l'évolution des pratiques et modes de création dont ceux afférents à l'usage des toponymes, d'autre part l'analyse du thème du voyage. Expérience dotée de multiples significations, le voyage rend sensibles le désarroi, le dénuement et la précarité. Ces sentiments se condensent dans l'expression emblématique kusa-makura 草枕, l'« oreiller d'herbe » qu'improvise le voyageur qui tente de trouver le repos en un lieu imprévu. L'auteure observe donc l'élaboration de 
conventions qui se constituent en tradition, ainsi que le renouvellement des formes de sensibilité et d'expression.

Dans la première partie, un travail de généalogie reconstitue à travers l'évocation d'antiques pratiques rituelles, puis de poèmes du Man.yō-sh $\bar{u}$, anthologie primordiale, le noyau originel de l'expression du voyage. Celui-ci est vécu comme "une douloureuse expérience d'arrachement» (p.15) due à la séparation, source d'une inépuisable nostalgie du pays et des êtres quittés. Un ton et une ambiance apparaissent, exprimés par des expressions récurrentes, ryojō 旅情, «sentiment du voyage», ryoshū 旅愁, “mélancolie du voyage», en lien avec cet autre terme emblématique aware 哀机, "émotion poignante ». Une figure du voyageur se constitue, celle d'un personnage mélancolique, voire pathétique, désorienté, diminué par l'absence de repères, flottant dans un monde mouvant. De façon symbolique, puis conventionnelle, ces états d'âme sont liés à l'automne, période de transition saisonnière qui aboutit au plus grand dépouillement après un moment d'éclat aussi splendide qu'éphémère.

Dès le Man.yō-shū, le voyage représente " autre chose qu'un simple déplacement » dans le territoire mais il est «avant tout, une expérience poignante de la fluctuation des choses » (p. 19). D'où l'importance du terme uku 浮 $<$, flotter, qui se développe en ukiyo 浮き世, sans doute dès l'origine compris comme à la fois «monde flottant » et « monde d'affliction ", superposant au registre sensible un sens moral. Se démultiplient les images de dérives dans le vent ou sur l'eau, d'éléments mouvants, insaisissables, instables comme la brume, les nuées, la clarté vague de la lune, les lueurs vacillantes, les barques ballotées sur les eaux. Le motif du nuage, qui recouvre nombre d'attributs, fait l'objet d'un développement dans le dernier chapitre du livre. La tonalité automnale ajoute ses notes tristes de vols d'oiseaux en partance, de brames solitaires, d'ondées glacées, de feuilles emportées, de rosée froide. Ainsi, paysages intérieur et extérieur se joignent, les phénomènes naturels traduisent l'intimité la plus profonde de l'être humain. Même l'éventuelle beauté perçue dans un site renvoie à sa solitude celui qui ne peut partager ce moment ou regrette de partir si vite. Rempli de franchissements éprouvants pour le corps et l'esprit, ce que souligne la topographie évoquée (pont, col, détroit, barrière,...), le voyage devient une "méditation sur la destinée humaine " (p. 30) et renvoie à une conception de la vie. Dans certains poèmes, persiste une valeur sacrée et propitiatoire destinée à apaiser les divinités des chemins, des passages et des lieux.

7 Ici se manifeste le statut littéraire particulier des toponymes, qualifiés à plusieurs reprises par Jacqueline Pigeot de "matériau poétique privilégié », et présents dans plus du tiers des poèmes du Man.yō-sh $\bar{u}$. Apparaît également un autre point qui nous semble important, « la proximité humaine instaurée à travers l'immensité du paysage » (p. 43). Dans la brève évocation du site, la présence de pêcheurs, d'une barque ou de tout autre écho du monde habité, reflet d'une activité vécue, donne l'échelle humaine dans un temps et un espace qui ont tendance à se dilater et à nous aspirer. Ces notations pointillistes rappellent notre appartenance à une " communauté de destin » et ancrent dans le monde. En surimpression à cette substance territoriale et temporelle qui semble parfois si ténue, les poètes vont reprendre inlassablement les images qui s'esquissent dans les poèmes du Man.yō-shū.

8 En résumé, cette première partie relève que le voyage constitue depuis longtemps un thème de prédilection et génère un ensemble d'images et de motifs récurrents. Des principes rhétoriques (mot-pivot kake-kotoba 掛詞, mot-appui makura-kotoba 枕詞, mot 
associé engo 縁語) se mettent en place afin de les articuler et de les organiser. Les éléments constitutifs du michiyuki-bun sont ébauchés dans les poèmes du Man.yō-shū : succession de noms de lieux, évocations descriptives des sites succinctes et dénuées de tout pittoresque, usage des possibilités offertes par les ressources phonétiques des toponymes, ambiance affective versée dans la mélancolie voire le désarroi. Ce que le michiyuki-bun va développer, ce sont des procédés de références et de citations, dispositifs qui se déploieront aussi dans les pratiques de l'espace.

9 La deuxième partie de l'ouvrage suit l'ordre chronologique et traite de la rhétorique des noms de lieux à l'époque classique. Nouvelle capitale au milieu d'un bassin, Kyōto est le centre politique et religieux du pays, et le palais impérial en est le foyer spirituel et culturel. S'y développent, entre autres, la poésie et les genres narratifs, s'y forment une sensibilité et un regard sur le monde, s'y élabore un système de références absolu qui va persister dans de nombreux domaines au-delà de cette période. Le monde extérieur est perçu en fonction de la hiérarchie des valeurs partagée par la noblesse de cour, société restreinte et étroitement liée qui construit et consolide ces références communes à travers ses pratiques sociales. Parmi celles-ci, la composition poétique et la diffusion de l'écrit sont essentielles. Les anthologies poétiques impériales constituent sans doute la forme la plus aboutie de sélection, d'agencement, et de présentation de ces valeurs et références, non dénuées d'ailleurs de signification politique.

La notion de meisho 名所, lieu renommé, apparaît également dans le Man.yō-shū. Si les noms de sites célèbres sont moins fréquents dans les anthologies poétiques de l'époque de Heian, la relation aux toponymes s'approfondit. La fréquence d'emploi permet à certains d'acquérir le statut d'uta-makura 歌枕, mot appui du poème. Quelles sont les caractéristiques de tels sites, réputés et mentionnés en poésie? Une typologie de ces lieux se dégage-t-elle ? Un meisho peut être connu par sa situation géographique le long d'une route : repère, comme un mont ou un massif montagneux, point de passage, comme un pont, une barrière ou un col, ou halte, comme un port ou une anse ; ou bien par son paysage caractérisé par un élément singulier : rochers jumelés dans la mer, baie parsemée d'îlots rocheux, langue de sable plantée de pins, lagune, lande, lac, rivière, relief particulier, arbre remarquable ; par sa valeur sacrée ou religieuse confirmée par la présence d'un sanctuaire ou d'un temple; par son rôle social important dû à sa fréquentation par la cour, en tant que lieu d'excursion ou de résidence séparée ; par son passé riche de faits historiques, légendaires ou mythiques ; par son aura littéraire ; par son nom même, source d'homonymies, de double sens, de lectures à clé. Ce qui fait la renommée, c'est le site, le nom du site, avec les événements qui s'y sont déroulés et la poésie qu'il a suscitée. C'est donc un ensemble de données matérielles et immatérielles à la croisée de plusieurs processus fondateurs: religieux, historique, politique, symbolique, esthétique.

11 Un site est d'autant plus remarquable qu'il combine plusieurs de ces caractéristiques, tout en se résumant par un ou deux motifs relevés dans son paysage, fragments fortement symboliques et chargés de références culturelles. Il accumule les empreintes d'une culture qui se densifie et en tire une force d'attraction, comme Yoshino, situé au sud de Nara, cité plus de mille fois parmi un corpus de 20000 poèmes de l'époque de Heian (p.68). Archétype fondamental chargé d'une part de mémoire collective, la puissance évocatrice de Yoshino sera même sollicitée lors de la création du site d'Ueno, à vocation de légitimation politique, dans la nouvelle capitale shogunale d'Edo au début du XVII ${ }^{e}$ siècle. Et c'est toujours à Yoshino que songe Tanizaki sous les cerisiers d'Ueno 
dans un passage très éclairant sur la persistance de ces processus accumulatifs et référentiels (p. 243-245).

Les plupart des sites célèbres se situent à proximité de la capitale de l'époque, Kyōto, et de la précédente, Nara, mais certains sont distribués à travers tout le pays, par exemple dans les provinces de l'est, le long de l'axe qui part de la capitale. Ils sont, en tout cas, rattachés à la même sphère culturelle, celle de la cour, qui concentre la majeure partie de l'activité poétique et littéraire. Les occasions de sorties sont nombreuses autour de la capitale, lors de fêtes, cérémonies, excursions, selon les saisons. Les pèlerinages connaissent un essor jamais démenti, mêlant toujours dévotion et curiosité récréative à des degrés variant selon la ferveur religieuse du pèlerin (et ses moyens financiers). La composition de poèmes qui accompagne la vie publique ou privée des membres de la noblesse, se poursuit pendant et après ces voyages où les sites ont pour "rôle de déclencher l'activité poétique » (p. 76).

Les concours de poèmes, nouvelle pratique de cour, en générant le concept de jugement, donc de critique, fournissent l'occasion d'établir la notion de sujet de composition et de formuler des critères esthétiques afin de fonder des comparaisons. Les sujets privilégiés sont semblables aux classements thématiques des anthologies impériales: d'abord les saisons, du printemps à l'hiver, l'amour, puis autres. Ces concours ont lieu à la cour, loin des sites, et les toponymes, si souvent sollicités, sont rarement sujets de poème. Ils sont choisis pour leurs ressources phonétiques, leur étymologie, leur puissance évocatoire en adéquation avec le sujet donné. De façon délibérée, les poètes ont "pris le parti d'évoluer dans le monde des mots, non dans celui des choses»(p.81). Cependant, même seulement suggérées dans un aperçu paysager qui matérialise un environnement, les choses ne disparaissent pas complètement. Un poème cité (p. 81) évoque la fameuse rivière Tatsuta qui entraîne les feuillages rougeoyants des momiji. Les composants donnés du paysage forment au sens littéral un authentique «lieu commun», reconnu et partagé par tous. S'institue une expression figurative du lieu qui irriguera tant de créations et formes artistiques, et deviendra, entre autres, un motif paysager intégré dans les jardins.

Ces données paysagères précises et concises sont issues sans doute d'un autre procédé de sélection et de façonnage d'après un fragment de nature. Les paysages miniatures reconstitués sur un plateau, nommés suhama 州浜, sont en vogue à la même époque. Les contraintes spécifiques à de telles compositions ont engendré des motifs, voire des signes conventionnels caractérisés par la miniaturisation. À travers ces procédés littéraires et ces dispositifs plastiques se confirme un processus de condensation des sites célèbres réduits à quelques éléments. Devenus emblématiques, ceux-ci constituent autant de motifs paysagers mis en œuvre de manières diverses, jouant avec les résonnances, les échos que les poèmes transmettent au cours du temps. Par exemple, à Edo, dans le jardin de Hamarikyū créé au XVII ${ }^{e}$ siècle, l'étang dont le niveau varie selon le rythme de la marée est une évocation du paysage de Shiogama. Son propriétaire faisait même extraire le sel dans des huttes fumantes pour ainsi rappeler un autre jardin disparu et mythique, le Kawara-no-in, et son créateur, Minamoto no Tōru. Le jardin, projection spatiale de surimpressions, évoque un paysage par des formes qui suscitent des émotions portées par la poésie. L'ici et le présent joignent l'ailleurs et le passé.

Un autre dispositif, qui est aussi une forme de médiation entre le monde matériel et les hommes, influe sur la perception et l'expression des sites célèbres : la confection de 
paravents ornés de peintures et de poèmes disposés dans des salles d'apparat. La succession des mois et les meisho relevant d'une longue tradition poétique sont les thèmes en faveur pour ces paravents, complémentaires car les mois sont caractérisés par une activité de saison se déroulant en un endroit donné, tandis que les lieux manifestent une ambiance saisonnière précise. Dans les sites célèbres, la spatialité s'accorde en effet, voire se subordonne, à la temporalité. Le rapport entre image et texte vise aussi la complémentarité, et non la simple illustration ou le commentaire, d'autant plus que poètes et peintres travaillent de façon indépendante et parallèle, à partir d'éléments acquis grâce aux œuvres préexistantes, devenus des conventions préalables et nécessaires. Jacqueline Pigeot en conclut que cette pratique des paravents peints « a certainement contribué à ancrer la notion de site célèbre dans l'imaginaire japonais » (p. 90).

Un système d'images se tisse, chacune en lien avec d'autres. "Ce que proposent les poètes, ce ne sont pas des images pittoresques, mais des cadres pour la rêverie" (p. 91). Un personnage s'exprime à travers un poème, ou bien est en marche dans la peinture, ce qui permet une forme d'identification. On peut se projeter soi-même dans une évocation paysagère qui est impulsion vers l'ailleurs plutôt que cadre fermé, laisser filer ses propres songes, souvenirs ou désirs, en résonnance avec les phénomènes naturels suggérés. Parfois, dans un processus d'identification empathique qui se dédouble, celui qui regarde la peinture ou lit le poème se reconnait dans ce voyageur qui lui-même se confond à un élément naturel du paysage, exprime ainsi une émotion, et sans doute aussi un sentiment d'appartenance au monde. Jacqueline Pigeot propose un bel exemple de ce processus (p. 92) avec un poème du Tsurayuki-shū : «Serais-je le seul / À leur demander abri ? / Non, les blanches vagues / Elles aussi, les harcèlent / Les sveltes pins du rivage ». Le poète qui "pense comme une vague » n'est pas sans évoquer Aldo Leopold (1887-1948), forestier et écologiste américain, acteur et penseur de l'éthique environnementale, revenu au premier plan aujourd'hui, qui voulait nous réapprendre à "penser comme une montagne » dans un texte de son Almanach d'un comté des sables (1949).

17 Par cette conception du paysage et de la nature qui s'élabore, les sites sont mis en avant non pour eux-mêmes, leur beauté pittoresque, mais pour ce qu'ils disent de notre humaine condition, celle de passant précaire en ce monde, de voyageur au cours de la vie. L'intention n'est pas de peindre un site pour lui-même en une miniature accomplie mais plutôt d'évoquer le regard que portent les humains sur lui. Le dessein des auteurs est de transmettre les sentiments et émotions suscités par une scène, d'éveiller notre empathie envers le témoin figuré dans la peinture ou qui énonce le poème. L'être humain est uni au paysage par un lien temporel, inséré dans le cycle saisonnier dont les moindres mutations sont attentivement observées, notées, reconnues par des conduites afférentes et une sensibilité partagée. De multiples pratiques se ritualisent et instituent un calendrier social qui suit le déroulement des saisons et s'inscrit dans des lieux consacrés subordonnés à celles-ci. Plus que la beauté des sites, ce qui importe, c'est leur capacité à capter et rendre perceptibles les mutations saisonnières continues, selon les critères qui sont élaborés par la tradition et modèlent l'appréciation de la nature.

$18 \mathrm{Au}$ cours de l'époque de Heian, les pratiques de cour ont fortement contribué à créer des relations aux lieux tout à fait spécifiques. Entre les sites et les humains viennent s'interposer diverses médiations : dispositifs tels que paysages miniatures ou paravents ornés de peintures et poèmes, ou bien procédés tels que rencontres poétiques. Ainsi, se 
sont établies des conventions, se sont formés des modes de lecture, d'interprétation et de transposition des sites, se sont construits des liens très élaborés avec le paysage et les faits de nature. Les sites célèbres, grâce notamment à un puissant processus de condensation, sont devenus éléments signifiants intégrés à la culture. Le site étant déjà contenu tout entier dans son nom, le travail des poètes peut alors essentiellement porter sur le toponyme. Plutôt que d'évacuation totale de la réalité, on pourrait peutêtre parler d'une réduction (dans le sens chimique où moins devient plus) au profit du nom associé à quelques éléments emblématiques (dont une étude typologique serait à faire). Le nom de chaque site est investi par un appareil rhétorique en cours d'élaboration dans lequel l'analogie a une part constitutive importante. On peut parler de codage et transcodage qui se superposent et que Jacqueline Pigeot n'hésite pas, parfois, à expliquer à l'aide d'exemples contemporains pris dans des registres populaires, le langage publicitaire par exemple.

19 À travers ces processus, certains toponymes vont être abandonnés, d'autres promus au rang d'uta-makura, mot appui du poème. Un resserrement géographique s'opère principalement dans l'aire autour de la capitale. La sélection se fait avant tout en fonction de la pertinence dans le champ rhétorique qui se met en place. Perdurent certains lieux formant la part immuable de l'héritage culturel. Ce tamisage se fait donc non en fonction des sites eux-mêmes, de leurs qualités physiques, mais surtout par rapport à leur nom, aux ambivalences phonétiques et aux possibilités d'associations qu'ils offrent, aux connotations qu'ils suggèrent. Ces propriétés permettent de démultiplier les liaisons ou glissements autour d'un toponyme, tout en associant un certain nombre d'éléments naturels qui mettent en scène saison, moment, végétal, animal, météores, et étayent un réseau de métaphores. Le toponyme conserve une capacité à s'incarner dans des formes, des sons, des faits de saisons, des composants naturels vivants qui permettent de surimposer une expression sensorielle à l'état affectif, comme la mélancolie du voyage. Le toponyme inscrit le texte dans le monde sensible, et par delà développe " une constellation d'images dont il est le centre ", " une charge de sens extralittéral » (p. 100), véritable "réalité augmentée ». "Introduire un nom de lieu célèbre dans un poème, c'est prendre en charge cette tradition, c'est donc s'insérer délibérément dans un réseau d'images et de correspondances » (p. 102), c'est le diffuser, l'enrichir et le consolider, se reconnaître dans cette culture et la partager.

Par la suite, les toponymes vont continuer à être enrichis d'associations nouvelles. La rigoureuse économie de moyens mise en œuvre, où chaque syllabe se sature de sens, démultiplie images, résonnances, émotions, suggère «le plus avec le moins de matière verbale » afin "d'accéder à l'au-delà de l'exprimé » propre à la poésie (p. 103). Aux waka, poèmes de trente et une syllabes qui subtilisent les effets de surimpression autour d'un seul toponyme, le michiyuki-bun va ajouter un effet d'enchaînement en liant plusieurs d'entre eux. Peut-être pouvons-nous mettre en parallèle ces formes de fluidité et de réticulation élaborées en littérature avec celles qui émergent dans les arts de l'espace. Architecture et jardin vont, peu à peu, mettre en œuvre de nouvelles relations entre dedans et dehors, des principes de condensation et miniaturisation, des dispositifs résorbant les distances dans l'espace ou le temps (comme le mitate 見立て, d'abord procédé littéraire), diverses formes de transitions et de juxtapositions de séquences spatiales. Dans le Mumyō-shō 無名抄, Kamo no Chōmei (1155 ?-1216) établit un parallèle entre l'art du jardinier et celui du poète, qui tendent tous deux à partir de fragments « à recréer la nature en vue d'un effet d'ensemble » (p. 129). 
21 Les toponymes bénéficient d'une place fondamentale dans la poésie japonaise, et cela depuis les plus anciennes compositions, ce que montre l'évolution du sens du mot utamakura, mot appui du poème. Son sens dérive vers "lieu célèbre chanté en poésie » après avoir désigné un manuel aide-mémoire utile à la pratique sociale de la poésie. Le nom d'un lieu célèbre est porteur en lui-même d'une puissance poétique et évocatoire, il introduit toute « une cristallisation d'images» (p. 134) qui appelle entre autres une forme, un paysage propres et non arbitraires. Les toponymes « sont essentiellement des foyers d'images, de réminiscences et d'émotions» (p. 355), donc de significations. A contrario, comme le dit clairement un commentaire lors d'un concours, l'absence de nom propre peut laisser un poème dans le vague, le trop peu suggestif, bref prive le texte de références, d'expressivité. L'économie de moyen devient banalité.

Ces réflexions trouvent un écho avec le fameux texte de Marcel Proust auquel Jacqueline Pigeot renvoie, Noms de pays : le nom (p. 132). À la différence que l'imaginaire nourri par les toponymes au Japon n'est pas personnel à un auteur mais partagé par tous les poètes et leur audience du fait de la rigueur dans le choix d'un nom propre existant, de son insertion dans la tradition, des références aux conventions communes. La tradition a tissé autour des toponymes un réseau d'images et de connotations, et cependant la véritable création dépasse la stricte conformité : "mots anciens, pensers nouveaux » exige le poète Teika (p.163). La pertinence d'un toponyme est jugée en fonction de sa conformité aux significations que la tradition poétique lui assigne, et la distanciation par rapport à la "réalité » fait aussi partie de cette convention. Les noms de lieux se sont détachés de leur substrat géographique (ou l'ont reconstruit) tandis que les substrats culturels et temporels se sont densifiés, leurs sens figurés, métaphoriques, connotatifs se sont enrichis. Ainsi «ils remplissent pleinement leur rôle de pourvoyeurs d'images" (p.142). Ils constituent une langue commune, véhiculent des images reconnues. La fonction du lieu célèbre à valeur d'uta-makura est d'ouvrir l'imaginaire poétique en évacuant la description, l'information, le pittoresque. Ce langage et cet imaginaire ont à leur tour (dans un mouvement trajectif comme le qualifie Augustin Berque) nourri la perception et la construction du territoire et certains de ces principes continuent à les irriguer aujourd'hui.

23 La plupart des poètes ne connaissent que par ouï-dire les toponymes qu'ils chantent, ils composent en chambre, voire revendiquent une indifférence totale aux sites réels. Ils nourrissent leur imaginaire dans la poésie, la puissance d'évocation des noms, s'appuient sur les conventions de la tradition, non pas dans la contemplation in situ - il semble que de manière générale, le «travail sur le motif » apparaisse en lien avec la modernité et une nouvelle conception du temps et de la vitesse. Cependant certains, comme Nōin (988-1050), Gyōson (1055-1135) et Saigyō (1118-1190), sont connus pour leurs nombreuses pérégrinations et sont même considérés comme des "fous de meisho » (p. 149) d'où ils ramenaient de précieux souvenirs à valeur de reliques (aiguille de pin, coquillage, et même une grenouille séchée), qu'ils montraient pieusement. Ce n'est sans doute pas un hasard s'ils sont aussi considérés parmi les plus grands poètes comme des légendes.

24 Au début de l'époque de Kamakura, apparaît un genre nouveau d'anthologie poétique, les collections de cent poèmes sur les sites célèbres, meisho hyakushu uta 名所百首歌. Dans un exemple présenté, les poèmes sont classés de façon traditionnelle par saisons, mais, est-il noté par l'auteure, sans cohérence géographique. Un autre recueil comprend, après les catégories thématiques classiques, une série de poèmes sur les 
meisho classés par types de paysage. Comme dans les lexiques poétiques, sont traités d'abord les paysages terrestres (montagnes, landes...) puis les paysages maritimes. Jacqueline Pigeot souligne toutefois l'absence d'itinéraire, de lien géographique entre ces différents lieux énumérés. Cependant cette lecture du territoire qui établit une typologie paysagère nous semble très intéressante. La géographie, ce n'est pas seulement la situation (sans importance dans ces exemples), mais c'est aussi le site et ses caractéristiques. Classer puis évoquer un lieu par son nom et par son paysage ancre celui-ci dans le territoire, non par sa situation, sa localisation, mais par la nature de son site, qui s'incarne en général en un composant ou un motif paysager saisi dans une mutation saisonnière.

La troisième partie de l'ouvrage traite des relations d'itinéraires proprement dites, à travers l'analyse de divers exemples. Depuis l'origine, les récits d'itinéraires ne sont pas conçus comme des narrations anecdotiques jalonnées de descriptions pittoresques, mais plutôt comme l'expression d'un mouvement qui superpose plusieurs dimensions temporelles : le temps horizontal de la marche du voyageur, de son parcours personnel qui se déroule; le temps vertical de l'épaisseur historique des lieux mentionnés, histoire transfigurée par la création littéraire. S'ajoute une dimension temporelle imaginaire, celle propre au poème, au moment de son énoncé ou de sa lecture. Les sites, à travers leur nom, sont des points de jonction où se croisent et se superposent ces différentes temporalités. Le michiyuki-bun retrace souvent l'itinéraire d'un personnage solitaire, victime d'un sort contraire, contraint à des pérégrinations dans des conditions pénibles, celles de l'exil, de la fuite, du bannissement ou de l'errance. Le registre affectif est souligné par un état fusionnel entre les sentiments de cet être pitoyable et le milieu qui l'entoure, ou plutôt dont il fait partie, comme un prolongement de lui-même. L'histoire des sites traversés se déchiffre à travers les poèmes qui l'ont cristallisée, ont conféré au lieu cette densité spécifique et continuent à susciter de nouveaux poèmes, ce qui amplifie l'effet de surimpression.

Le propre des meisho est d'être "éveilleurs de fantasmes» (p. 244). Par exemple, contempler la lune dans un lieu renommé touche davantage, prend une substance différente, bien plus riche et intéressante qu'en un endroit quelconque. Mais sont-ils interchangeables, comme se demande Jacqueline Pigeot, car leur réalité physique compte si peu en regard de l'image qui s'en est construite? L'auteure par ailleurs remarque l'homophonie des deux verbes nagamu, contempler vers le lointain 眺む et aussi dire un poème 詠む, comme si la contemplation des sites et la récitation poétique étaient deux pratiques quasi équivalentes, liées, voire indissociables dans un certain rapport au monde. Réciter un poème aurait valeur de contemplation, de visite virtuelle, sans avoir besoin de se déplacer, ce qui comporte toujours des risques. «Dans l'espace comme dans le temps, le meisho est avant tout un lieu où s'inscrit l'imaginaire collectif, où se déploie, en accord avec lui, la rêverie personnelle» (p. 245). De plus, aux nombreuses réminiscences culturelles que les lieux inspirent, collectives et transcrites dans la littérature, peut s'ajouter le ressouvenir personnel.

Les principales caractéristiques du michiyuki-bun sont, d'une part, la fusion de la prose narrative et des poèmes, et d'autre part, l'introduction de la citation poétique, l'assimilation de la fonction de référence. Des fragments de poèmes anciens, témoins et véhicules de la tradition, sont incorporés au texte ainsi que de nouvelles compositions suscitées par le passage en un lieu célèbre du voyageur au cours de son itinéraire. Le michiyuki-bun atteint un effet de continuité temporelle et spatiale très fort, au travers 
de l'enchâssement de l'héritage littéraire et de l'enchaînement des toponymes, porteurs déjà d'une longue tradition.

La quatrième partie, fertile étude thématique, aborde "représentation du voyage et poétique du michiyuki-bun ». De multiples images, motifs récurrents, formes narratives, qui donnent cette tonalité poignante à de nombreux textes, sont issus de cette conception du voyage propre au Japon ancien et déjà évoquée. Un récit mythique s'est constitué en schéma narratif qui persiste et répète l'itinéraire d'un personnage de haute condition en marche vers la mort. Son parcours symbolique enchaîne d'une façon rituelle transgression / expulsion / purification / régénérescence qui s'incarnent en un itinéraire. À l'origine, le cheminement de la divinité mythologique Susanoo-no-mikoto, premier d'une longue postérité, constitue l'archétype de ce modèle culturel qui va évoluer, amplifier ses significations, parfois politiques, par exemple dans le cas d'aristocrates exilés, voire écrasés. La situation du banni s'auréole peu à peu d'une sorte de prestige et suscite des sentiments de compassion, d'affectivité; elle est valorisée par une forme de "délectation esthétique» (p. 299). C'est à travers les expressions littéraires de ces histoires, leur sublimation, qu'une "conception du voyage comme expérience à la fois pathétique et valorisante» (p.302) se construit, "symbole de la précarité non seulement sociale, mais aussi morale, de l'être humain » (p. 303). Le personnage de la femme littéralement «folle de chagrin» (p. 307) présent dans de nombreuses pièces de Nō, en quête éperdue d'un être cher, confirme "la transmutation de la détresse humaine en objet d'émotion et de contemplation esthétique » (p. 311).

Dans l'évolution de cette sensibilité, le bouddhisme semble déterminant à plus d'un titre: les moines, souvent eux-mêmes voyageurs, ont laissé des écrits importants et leurs pérégrinations sont empreintes de multiples significations et valeurs. Le pèlerinage en est la forme par excellence, réunissant «exercice à valeur religieuse, contemplation mystique de la nature, activité poétique» (p.313). Au contact de certains paysages au rôle de "stimulant spirituel», l'extase devant la nature et la méditation mystique fusionnent, " la ferveur religieuse se confond avec l'imagination poétique de la nature " (p.317). Le voyage, parcours et initiation, est donc à la fois «métaphore et mise en pratique de la progression spirituelle» (p.322), voie vers le détachement. La contemplation au contact $d u$ monde naturel amène à connaître l'intrinsèque fragilité des choses, à déchiffrer la loi d'impermanence, aboutit même à l'identification avec les éléments naturels. Occasion de saisir l'écoulement du temps, le voyage amène aussi à vivre en accord avec la nature, au rythme des moments, des saisons. Mais parfois l'angoisse de l'inconnu, la mortification, surgissent, et alors c'est toute la vie qui ne semble qu'une pérégrination entre deux étapes, la naissance et la mort. Au final, « le voyage n'enseigne que la vanité du voyage » (p. 332). Expérience du temps et expérience de l'espace s'articulent non pas comme déploiement par l'homme de sa liberté mais plutôt comme «flottement générateur d'angoisse » (p. 334).

Le voyage et la poésie sont donc vécus de façon complémentaire et cette double expérience s'appuie sur la notion de meisho. Leur compréhension est, au Japon, une expérience culturelle élaborée qui permet de connaître, partager et renouveler émotions, sensations, valeurs esthétiques et spirituelles. Étape et relais où se succèdent les générations, « butte-témoin » dont la pérennité révèle en miroir la brièveté de notre passage, un meisho peut pourtant disparaître. Dans ce cas, la poésie demeure seule trace d'une rencontre passée entre un site et des hommes « comme la lumière visible d'une 
étoile pourtant refroidie » (p. 350). Cette comparaison nous évoque immédiatement la Sumida, longtemps unique meisho consacré uta-makura de la cité d'Edo, étoile en train de s'éteindre dans le Tōkyō du début du $\mathrm{xx}^{\mathrm{e}}$ siècle dont Nagai Kafū nous a transmis les dernières lueurs. partir de l'analyse d'un texte exemplifié, un poème à chanter du XII ${ }^{\mathrm{e}}$ siècle, Kaihen 海辺, "Bords de mer». Le contenu référentiel se rapportant à l'espace, au temps, aux événements disparait au profit de la connotation, du symbolique. La notion d'étape, c'est-à-dire d'organisation et de découpage dans le temps et l'espace, est disloquée « au profit du Temps comme succession de phénomènes sensibles, (...) comme mutation dans laquelle l'homme est physiquement impliqué » (p.362). "Le réel éclate » en un " poudroiement d'images fugitives ", en fragments labiles qui s'enchaînent, ou plutôt se juxtaposent, dans un flux continu et rythmé qui "charrie» citations, évocations, allusions, démultipliant l'effet de miroitement. La recomposition de l'itinéraire disloqué s'opère par la continuité et la régularité du rythme de l'écriture. Dans ces paysages si peu décrits, si peu représentés mais évoqués par quelques notations qui expriment une atmosphère - «le vrai poète est celui qui peint non pas les réalités (keibutsu 景物) mais le halo qui les entoure (omokage 面影)》 expose Shinkei au $\mathrm{Xv}^{\mathrm{e}}$ siècle dans un traité (p.365) - en continuo une figure est déclinée. C'est celle de la présence humaine notée par des activités (navigation, pêche, ramassage de coquillages ou d'algues...) qui habitent ces paysages perçus de façon très polysensorielle.

Au cours de son histoire, le Japon, c'est-à-dire ses territoires et sa nature, a été travaillé par la culture, foncièrement par la poésie. Les myriades de poèmes, chargés de mythes, de symboles, d'images sans cesse ressassées, ont modelé les toponymes, "sceaux de l'homme sur l'espace» (p.371) et construit une topographie imaginaire toujours rémanente dans la mémoire collective. Par son travail, Jacqueline Pigeot nous permet de comprendre et sentir comment s'est constitué ce réseau vivant des sites célèbres, essentiels aux pratiques littéraires aussi bien qu'à celles de l'espace : lieux à la fois réels et idéels; lieux vécus et visités, ou bien rêvés et évoqués de multiples façons, par la poésie puis par différents modes d'expression comme l'art des jardins; lieux où les temps se superposent et fusionnent avec l'espace.

À travers la notion hétérotopique de meisho, ce sont des relations de la société japonaise à son territoire, à ses milieux naturels, à ses saisons, ce sont des articulations de la culture au temps et à l'espace qui se sont construites. L'étude de Jacqueline Pigeot nous montre comment des lieux se sont particularisés, puis ont servi d'appui à un imaginaire poétique qui à son tour a informé divers pratiques et modes de créations. Ce livre est donc précieux et utile pour tous ceux qui, comme nous, s'intéressent au sens de l'espace au Japon, qui voudraient mieux comprendre la production des références fondatrices de son imaginaire paysager. C'est cette approche qui a guidé notre lecture. 